\title{
RANK, RANKL and osteoprotegerin in arthritic bone loss
}

\section{M.C. Bezerra, \\ J.F. Carvalho, \\ A.S. Prokopowitsch and R.M.R. Pereira}

\author{
Departamento de Reumatologia, Faculdade de Medicina, Universidade de São Paulo, \\ São Paulo, SP, Brasil
}

\author{
Correspondence \\ R.M.R. Pereira \\ Departamento de Reumatologia \\ FM, USP \\ Av. Dr. Arnaldo, 455, Sala 3107 \\ 01246-903 São Paulo, SP \\ Brasil \\ Fax: + 55-11-3066-7490 \\ E-mail: rosamariarp@yahoo.com or \\ reumato@edu.usp.br
}

Publication supported by FAPESP.

$\ldots \ldots \ldots \ldots \ldots \ldots \ldots$

Received May 10, 2004

Accepted December 9, 2004 ..................

\begin{abstract}
Rheumatoid arthritis is characterized by the presence of inflammatory synovitis and destruction of joint cartilage and bone. Tissue proteinases released by synovia, chondrocytes and pannus can cause cartilage destruction and cytokine-activated osteoclasts have been implicated in bone erosions. Rheumatoid arthritis synovial tissues produce a variety of cytokines and growth factors that induce monocyte differentiation to osteoclasts and their proliferation, activation and longer survival in tissues. More recently, a major role in bone erosion has been attributed to the receptor activator of nuclear factor kappa B ligand (RANKL) released by activated lymphocytes and osteoblasts. In fact, osteoclasts are markedly activated after RANKL binding to the cognate RANK expressed on the surface of these cells. RANKL expression can be upregulated by bone-resorbing factors such as glucocorticoids, vitamin D3, interleukin 1 (IL-1), IL-6, IL-11, IL-17, tumor necrosis factor- $\alpha$, prostaglandin $E_{2}$, or parathyroid hormone-related peptide. Supporting this idea, inhibition of RANKL by osteoprotegerin, a natural soluble RANKL receptor, prevents bone loss in experimental models. Tumor growth factor- $\beta$ released from bone during active bone resorption has been suggested as one feedback mechanism for upregulating osteoprotegerin and estrogen can increase its production on osteoblasts. Modulation of these systems provides the opportunity to inhibit bone loss and deformity in chronic arthritis.
\end{abstract}

Key words

- Osteoprotegerin

- RANK/RANKL

- Arthritis

- Bone loss

- Erosion 
CSF), tumor necrosis factor alpha (TNF- $\alpha$ ), interleukin-1 (IL-1), and parathyroid hormone-related peptide are known to induce monocyte/macrophage differentiation to osteoclasts. The major factor responsible for osteoclast cell differentiation has been cloned and identified as receptor activator of nuclear factor kappa B ligand (RANKL) (4-6).

\section{Receptor activator of nuclear factor kappa B ligand}

The rankl gene encodes a TNF superfamily molecule, RANKL of 316 amino acids (38 kDa) plus three RANKL subunits form the functional molecule. It is formed as a membrane-anchored molecule and can then be released from the cell after proteolytic cleavage by the metalloprotease desintegrin TNF- $\alpha$ convertase (7). RANKL is highly expressed on osteoblast/stromal cells, primitive mesenchymal cells surrounding the cartilaginous anlagen and hypertrophying chondrocytes (4). In addition to playing a role in the differentiation of osteoclasts from their precursor cells, RANKL also promotes increased activity and survival of these cells by an anti-apoptotic effect $(4,7)$.

There are transgenic animal models including knockout of the rankl gene, which develop severe osteopetrosis (8). These animals have a complete block in osteoclast development that can be restored after the reintroduction of the rankl gene into bone marrow progenitor cells (9).

\section{Receptor activator of nuclear factor kappa B}

The receptor activator of nuclear factor kappa B (RANK) is a member of the TNF receptor superfamily expressed on the surface of hematopoietic osteoclast progenitors, mature osteoclasts, chondrocytes, mammary gland epithelial cells, and trophoblast cells $(10,11)$. It is a transmembrane heterotrimer containing three molecular in- tracellular domains (I, II and III). In vitro, binding of RANK with its cognate RANKL results in osteoclastogenesis by monocyte/ macrophage progenitor differentiation to osteoclasts and the activation of mature osteoclasts (11).

Activation of the RANK receptor on the osteoclast surface triggers intracellular signals mediated by the interaction of intracellular I, II and III domains and adapter proteins, TNF receptor-associated factors (TRAF). These TRAF-binding domains of the RANK molecule are important for the RANK-dependent induction of nuclear factor kappa $\mathrm{B}$ and c-Jun $\mathrm{NH}_{2}$-terminal kinase activities. RANKL also activates the anti-apoptotic serine/threonine kinase Akt/PKB through a signaling complex involving c-Src and TRAF6. c-Src and TRAF6 interact with each other and with RANK after receptor engagement and deficiency of c-Src or addition of Src family kinase inhibitors blocks RANK-mediated Akt/PKB activation in osteoclasts (12).

\section{Osteoprotegerin}

Osteoprotegerin $(\mathrm{OPG})$ is a protein with homology to members of the TNF receptor family and is produced and released by activated osteoblast cells (13). OPG functions as a soluble decoy receptor for RANKL and competes with RANK for RANKL binding. Consequently, OPG is an effective inhibitor of osteoclast maturation and osteoclast activation in vitro and in vivo $(8,13)$.

High systemic levels of OPG in OPG transgenic mice cause osteopetrosis, as also observed in rankl knockout mice. As expected, OPG-deficient mice display severe osteoporosis associated with a high incidence of fractures, indicating that the level of bone mass correlates with the levels of OPG in mice (14).

The balance between RANKL-RANK signaling and the levels of biologically active OPG regulates development and activation 
of osteoclasts and bone metabolism. All factors that inhibit or increase bone resorption via osteoclasts act via regulation of RANKLRANK and/or OPG-RANKL interactions (Figure 1).

\section{RANK/RANKL/OPG and the immune system}

In addition to its role in osteoclast development, RANKL was found to be expressed in activated T-cells, lymph nodes, spleen, thymus, intestinal lymphoid patches, and immature CD4/CD8 thymocytes (5,6). RANK is expressed on the surface of dendritic cells, mature T-cells and hematopoietic precursors, and its interaction with RANKL can induce $\mathrm{Bcl}-\mathrm{X}_{\mathrm{L}}$ expression, CD40 expression and IL-12 production in dendritic cells; moreover, RANK/RANKL interaction can produce proliferation of T-cells activated by dendritic cells $(5,6,16)$. In contrast to the CD40/CD40L system, RANK/RANKL signaling does not affect the expression of surface molecules, and maximum levels of RANKL are attained $48 \mathrm{~h}$ after the initial activation of T-cells (and sustained for $96 \mathrm{~h}$ ), while $\mathrm{CD} 40 \mathrm{~L}$ is rapidly expressed and downregulated (17). This suggests that CD40/ CD40L interactions might control the initial phases of the response, while RANK/RANKL might act at later time points. RANK and RANKL are also important factors in lymphoid tissue development and in maturation of $\mathrm{T}$ - and B-cell precursors in bone marrow.

Like RANK, OPG can be found on the surface of dendritic cells. It has been suggested that RANK/RANKL interactions might regulate dendritic cell functions, T-cell activation and T-cell/dendritic cell communication in vitro (5), and it is possible that OPG modulates these interactions, participating in the regulation of these phases of the immune response. OPG decreases the production of cytokines (IL-6 and IL-11) in response to dendritic cell stimulation by RANKL, and decreases the generation of cytokines (IL-12

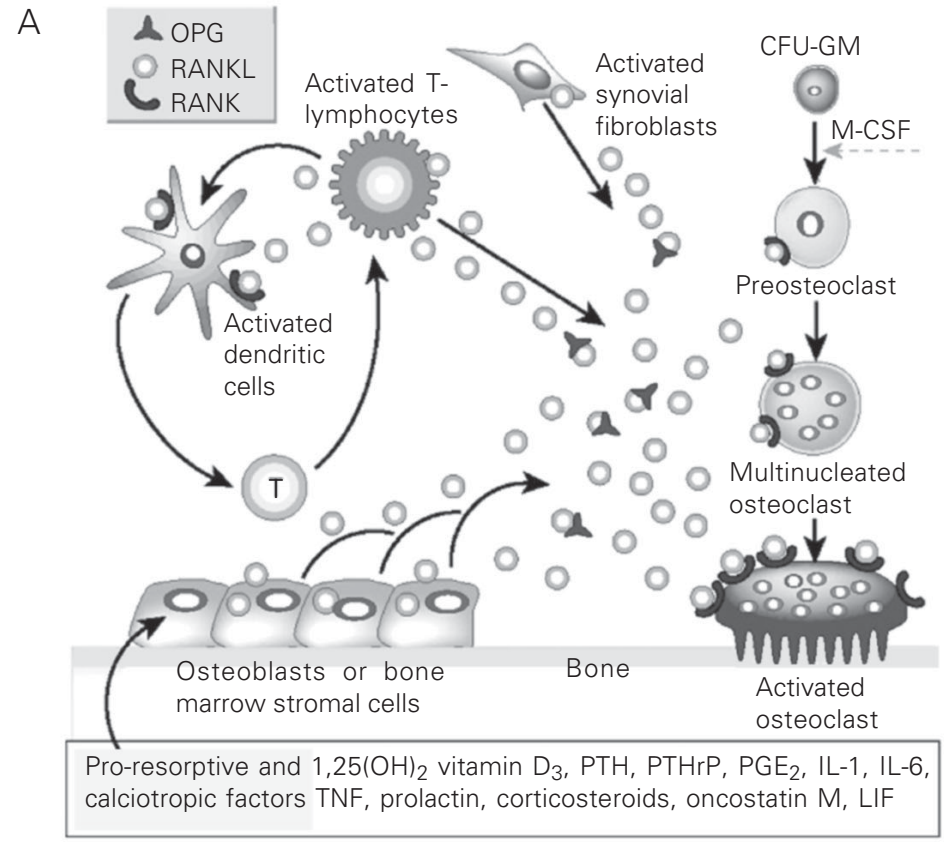

B

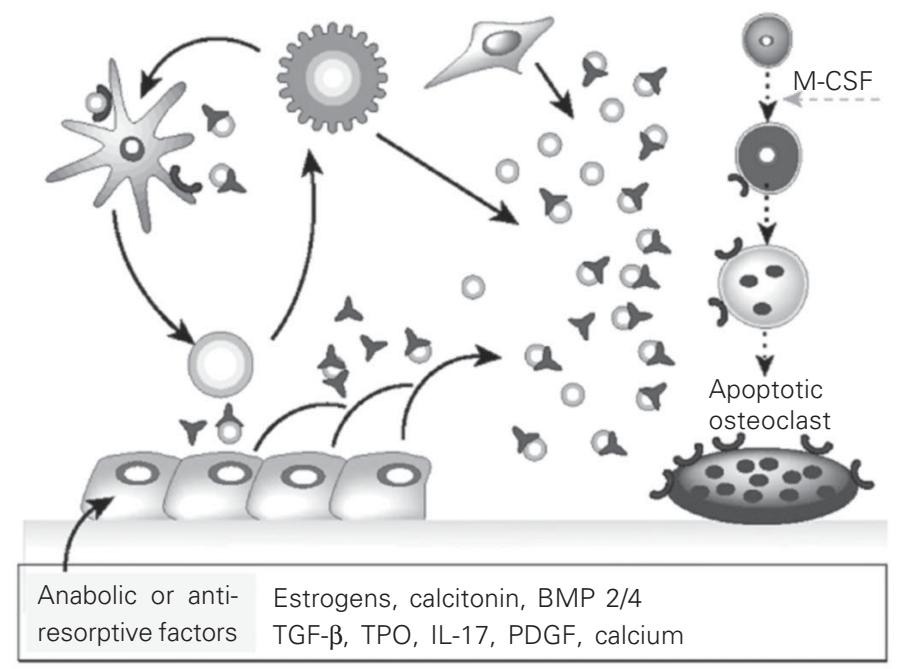

Figure 1. Hormonal control of bone resorption. Schematic representation of the mechanism of action of $A$, pro-resorptive and calcitropic factors and $B$, anabolic and antiosteoclastic factors. Receptor activator of nuclear factor kappa B ligand (RANKL) expression is induced in osteoblasts, activated T-cells, synovial fibroblasts and bone marrow stromal cells, and RANKL subsequently binds to its specific membrane-bound receptor activator of nuclear factor kappa B (RANK), thereby triggering a network of tumor necrosis factor (TNF) receptor-associated factor-mediated kinase cascades that promote osteoclast differentiation, activation and survival. Conversely, osteoprotegerin (OPG) expression is induced by factors that block bone catabolism and promote anabolic effects. OPG binds and neutralizes RANKL, leading to a block in osteoclastogenesis and decreased survival of pre-existing osteoclasts. CFU-MG = colony-forming units-granulocyte-macrophage; $\mathrm{M}$ $\mathrm{CSF}=$ macrophage colony-stimulating factor; $\mathrm{PTH}=$ parathyroid hormone; $\mathrm{PTHrP}=\mathrm{PTH}-$ related peptide; $\mathrm{PGE}_{2}$ = prostaglandin $\mathrm{E}_{2} ; \mathrm{IL}=$ interleukin; $\mathrm{LIF}=$ leukemia inhibitor factor; BMP $2 / 4=$ bone morphogenetic proteins 2 and $4 ; \mathrm{TGF}-\beta=$ transforming growth factor $B$; $\mathrm{TPO}=$ thrombopoietin; PDGF $=$ platelet-derived growth factor. (Reproduced, with permission, from Ref. 15). 
and IL-15) by proliferating T-cells (18).

It has been shown that RANKL can increase the number and persistence of antigen-presenting dendritic cells in vivo (16). Moreover, RANKL treatment increases antigen-specific primary $\mathrm{T}$-cell responses and appears to be an important factor in memory T-cell responses (16), probably by increasing and/or changing the production of cytokines such as IL-12. However, none of these molecules (RANKL, RANK, OPG) seems to play an essential role in T-cells or dendritic cells that cannot be compensated for by other molecules such as CD40/CD40L, although they can influence some aspects of lymphocyte and dendritic cell functions (19). The expression of these molecules can be influenced by sex hormones $(10,20)$, so it is possible that they control sex-specific differences in immunity and could be involved in the higher incidence of some autoimmune diseases in women, for example. Surprisingly, in vivo studies have shown T-cells to be crucial to the mechanism by which estrogen deficiency induces bone loss. T-cells from ovariectomized mice produce increased amounts of TNF which augments RANKL. Roggia et al. (21) showed that ovariectomy induced rapid bone loss in wild-type mice but failed to do so in TNF-deficient mice. Thus, the true function of RANK/RANKL/OPG in the immune system remains to be elucidated.

Several cytokines (TNF- $\alpha$, IL-1, IL-11, IL-17, and others) with regulatory effects on immune function appear to contribute to bone homeostasis by enhancing bone resorption. These cytokines have already been identified in the rheumatoid synovium and may promote osteocartilaginous resorption by stimulating osteoclastic mediators (22). Synovial tissue provides a source of RANKL that could influence osteoclastogenesis. Furthermore, synovial fibroblasts and T-lymphocytes (3) from patients with RA produce mRNA and protein (2) for RANKL. Synovial tissues may also provide a source of osteoclast precursor cells since macrophages iso- lated from RA differentiate into osteoclasts in the presence of M-CSF plus RANKL. Cells digested from RA synovial tissue samples generate osteoclasts, tartrate-resistant acid phosphatase (TRAP)-positive multinucleated cells that form resorption pits on dentin slices. The formation of these resorption pits is inhibited by OPG. In addition, the number of resorption pits strongly correlates with the ratios of RANKL/OPG mRNA levels. Given the important role of RANK, RANKL and OPG in bone metabolism and immune function, it has been suggested that the RANK/ RANKL/OPG system and these cytokines may work together in order to cause bone resorption via regulation of the RANKL/OPG ratio (23). Several lines of evidence have shown that some of these cytokines can influence the expression of RANKL and OPG. For example, IL-1, IL-11, IL-17, TNF$\alpha, P T H r P$, and prostaglandin $\mathrm{E}_{2}$ can increase RANKL mRNA expression by T-cells, and PTHrP and prostaglandin $\mathrm{E}_{2}$ can decrease OPG mRNA expression in these cells $(22,24)$. Moreover, IL-6 can induce RANKL mRNA expression in cultures of stromal cells from rodents (25), and IL-1 and TNF- $\alpha$ can cause stromal cells and osteoblasts to produce IL7 , which induces osteoclastogenesis via the RANKL system (26).

IL-17 is a crucial cytokine for osteoclastic bone resorption and may participate in the development of cartilage destruction in RA patients $(27,28)$. The levels of this cytokine were significantly higher in RA patients and anti-IL-17 antibody significantly inhibited osteoclast formation induced by culture medium of RA synovial tissues. IL-1 is another important cytokine involved in synovial inflammation and bone resorption. Chabaud and Miossec (29) demonstrated that the combination of TNF- $\alpha$, IL- 1 and IL17 receptors was more effective in inhibiting bone resorption than each cytokine alone in RA synovium and bone explants.

It has also been demonstrated that peripheral blood mononuclear cells from patients 
with psoriatic arthritis have a marked increase in osteoclast precursors and readily form osteoclasts in vitro without exogenous RANKL or M-CSF (30).

However, a counter-regulatory mechanism by which activated T-cells can inhibit the osteoclastogenesis induced by RANKL has been recently described (31). This mechanism involves the participation of interferon- $\gamma$ (IFN- $\gamma$ ), which interferes with the signal generated by RANKL. IFN- $\gamma$ reduces TRAF6 expression in a selective manner, causing an inhibition of the transcription pathways induced by RANKL and, therefore, decreasing osteoclast formation.

It has already been demonstrated that production of RANKL by activated T-cells can directly control osteoclastogenesis and bone remodeling in vitro and in vivo, and these effects can be blocked by the administration of OPG (16). So, systemic activation of T-cells can lead to bone loss, indicating that $\mathrm{T}$-cells are important mediators of bone loss in vivo. T-cells are probably not required for normal bone homeostasis, since mutant mice lacking $\mathrm{T}$-cells still have normal bone cavities and tooth eruption (32). However, it seems that chronic activation of $\mathrm{T}$ cells can affect bone remodeling by RANKL production and chronic glucocorticoid administration can lead to bone loss, probably by inducing RANKL expression and decreasing OPG production (33). These findings provide a framework for local or systemic bone loss associated with diseases that involve the immune system. Inhibition of RANKL function via OPG might prevent bone destruction in these diseases.

\section{RANKL and arthritis}

Focal or diffuse bone loss represents a major unsolved problem in RA. This is a condition of gradual joint destruction related to chronic inflammation with T-cell activation. The skeletal complications of RA consist of focal bone erosions and periarticular osteoporosis at sites of active inflammation and generalized bone loss with reduced bone mass. Local bone loss in the affected joints frequently results in life-long crippling. In this disease characterized by both inflammation and bone destruction, interactions between the RANKL/RANK/OPG system and T-cells may partly explain the lesions.

Arthritis in humans and in animal models is characterized by synovial inflammation, erosion of bone and cartilage, severe joint pain, and ultimately life-long crippling. For example, in Lewis rats, experimental induction of arthritis by subcutaneous injection of bacterial products in an adjuvant leads to severe inflammation of bone marrow and of the soft tissues surrounding joints, accompanied by extensive local bone and cartilage destruction, loss of bone mineral density, and crippling (34). In rats, this condition, called adjuvant-induced arthritis, mimics many of the clinical and pathologic features of human RA.

RANKL expressed on activated T-cells can trigger osteoclast activation and it is possible that RANKL/RANK might play a major role in inflammation-induced bone loss and joint destruction in arthritis (35). In the adjuvant-induced arthritis model, RANKL protein is expressed on the surface of synovial effector T-cells isolated at the clinical onset of arthritis. Inhibition of RANKL via OPG had no effect on the severity of inflammation. However, OPG treatment completely abolished the loss of mineral bone density in the inflamed joints of these animals in a dosedependent manner. Histologically, OPGtreated arthritic rats exhibited minimal loss of cortical and trabecular bone, whereas untreated arthritic animals developed severe bone lesions characterized by partial to complete destruction of cortical and trabecular bone, and erosion of the articular cartilages. These results showed that RANKL is a key mediator of joint destruction and bone loss in adjuvant arthritis. Importantly, whereas untreated rats experienced severe crippling, rats 
treated with OPG at the onset of the disease did not show any signs of clinical crippling.

Recent dosing data obtained for male Lewis rats showed that OPG preserves articular bone in arthritic joints in a dose- and schedule-dependent manner, halts bone erosion when given at any point during the course of arthritis, and produces sustained anti-erosive activity after a short course (36). Moreover, a single OPG bolus subcutaneously injected at the onset of the disease eliminated osteoclasts, preserved bone mineral density for 7 days, and prevented bone erosions for 4 days. No OPG dosage or regimen alleviated weight loss, inflammation, or periosteal osteophyte production. These data indicate that a single OPG injection will inhibit joint erosions for several days, and that OPG treatment is most effective in protecting joint integrity when initiated early during the disease (37).

Alteration of cartilage structures leading to cartilage collapse constitutes a critical step in arthritic joint destruction. In untreated arthritic rats, partial or complete erosion of the cartilage is observed in both the central and peripheral regions of joint surfaces. In striking contrast, neither cartilage erosion nor matrix degeneration in the centers of joint surfaces occurred in OPG-treated animals $(35,36)$. OPG could protect the cartilage by maintaining the overlying cartilage from the inflammatory cell infiltrates in the bone marrow. RANK, RANKL and OPG expression have been recently observed in normal cartilage (38). The functional relevance of RANKL-RANK expression in chondrocyte physiology is not known. Thus, in adjuvantinduced arthritis inhibition of RANKL activity by OPG can prevent cartilage destruction, a critical, irreversible step in the pathogenesis of arthritis.

Arthritis can occur in the absence of Tcells (39). Using in situ hybridization of inflamed rat joints and isolation of different cell populations from these joints, Kong et al. (35) demonstrated that RANKL is indeed expressed in lymphocytes, macrophages, and especially in synoviocytes. Similarly, genetic ablation of RANKL also does not prevent inflammation in an antibody-mediated model of arthritis using the $\mathrm{K} / \mathrm{BxN}$ serum transfer model (40). Multinucleated TRAP-positive osteoclast-like cells were abundant in resorption lacunae in areas of bone erosion in arthritic control mice, and were completely absent in arthritic rankl knockout mice, demonstrating the absolute requirement for RANKL in osteoclastogenesis in this serum transfer model of inflammatory arthritis (41). Cartilage damage was still observed in both arthritic rankl knockout mice and arthritic control mice but a trend toward milder cartilage damage in the rankl-1mice was noted. Thus, it appears that RANKL is not required for cartilage destruction but clearly plays a still unidentified modulatory role (41).

IL-1 and, to a lesser extent, TNF- $\alpha$ are critical mediators of antibody-induced arthritis in the serum transfer model (42). Importantly, inhibition of RANKL via OPG at the onset of disease prevents bone erosion and has a beneficial effect on cartilage destruction without affecting inflammation in a TNF- $\alpha$-induced arthritis model $(43,44)$. Furthermore, a significant reduction of osteoclast numbers was seen in animals treated with OPG. TNF- $\alpha$-triggered joint destruction is critically dependent on RANKL expression and OPG alone or in combination with bisphosphonates is an effective therapeutic tool for the prevention of TNF-mediated destruction of bone by reducing the number of bone-resorbing cells in the inflamed tissue (44).

Further evidence for the role of osteoclasts in bone erosion in arthritis comes from a recent study using the TNF- $\alpha$ transgenic mouse model in which mice develop a spontaneous, destructive polyarthritis at an early age (43). Osteoclast-targeted therapies were used to treat TNF- $\alpha$ transgenic mice, and inflammation, loss of bone and tissue de- 
struction were evaluated (44-46). Schett et al. (45) investigated systemic bone changes in human TNF transgenic mice which spontaneously developed severe inflammatory arthritis and osteoclast blockage with OPG. Osteodensitometry revealed a decrease in trabecular bone mineral density $(-37 \%)$ and histomorphometry revealed a dramatic loss of bone volume (-85\%) compared with wildtype controls. OPG completely blocked TNF$\alpha$-mediated bone loss by increasing bone mineral density $(+89 \%)$ and bone volume $(+647 \%)$. Zwerina et al. (46) investigated the efficacy of single and combined blockade of TNF, IL-1 and RANK pathways on synovial inflammation, bone erosion and cartilage destruction in a TNF-driven arthritis model. Synovial inflammation was inhibited by antiTNF $(-51 \%)$, but not by IL-1Ra or OPG monotherapy. The combination of anti-TNF with either IL-1Ra $(-91 \%)$ or OPG $(-81 \%)$ was additive and almost completely blocked inflammation. Bone erosion was effectively blocked by anti-TNF (-79\%) and OPG (-60\%), but not by IL-1Ra monotherapy. The combination of anti-TNF and IL-1Ra, however, completely blocked bone erosion $(-98 \%)$ and was the most effective double combination therapy in preventing cartilage destruction $(-80 \%)$.

RANKL is also expressed in collageninduced arthritis in which focal collections of osteoclasts are prominent at sites of bone destruction (47). After induction of collagen-induced arthritis in Dark Agouti rats, short-term OPG treatment at the onset of the disease effectively prevented joint destruction, even though it had no impact on the inflammatory aspects of collagen-induced arthritis. In arthritic joints, OPG treatment depleted osteoclast numbers by over $75 \%$ and diminished bone erosion scores by over $60 \%$. Although cartilage loss was also reduced by OPG, the effects on cartilage were again lower than those on bone (48). Similar to this rat model, it has been recently suggested that the RANKL-RANK system plays an important role in osteoclastogenesis in both local and systemic osteolytic lesions in autoimmune type II collagen-induced arthritis in mice (49). These studies provide evidence for the role of osteoclasts (and of RANKL) in the pathogenesis of bone erosion in arthritis in several animal models of arthritis with distinct pathogenetic mechanisms.

To extend these rodent studies to human patients with arthritis, inflammatory cells were collected from the synovial fluids of patients with adult or juvenile RA and patients with osteoarthritis and OPG and RANKL expression were evaluated (35). All RA patients and patients with advanced osteoarthritis tested $(\mathrm{N}>40)$ in this study exhibited RANKL expression in inflammatory cells while OPG expression was not detectable. In subsequent studies it has been shown that activated synovial T-cells, monocytes, and synovial fibroblasts express both membranebound and soluble forms of RANKL (50) and activated human T-cells expressing RANKL can induce osteoclastogenesis from autologous peripheral monocytes that can be blocked by dose-dependent inhibition with OPG (50). RA patients exhibit high serum levels of OPG and soluble RANKL, which normalize after anti-TNF- $\alpha$ treatment (51). RANKL mRNA is present in cells isolated from the pannus and synovial membrane regions of RA patients (52). Conversely, RANKL is not present in normal synovium, suggesting a link between the expression of RANKL mRNA and the development of rheumatoid synovial lesions (53). Osteoclasts have also been identified at sites of maximum RANKL expression, i.e., at the pannus-bone interface $(1,54)$. Recent data have indicated a major role for osteoclasts in rheumatoid bone erosions $(2,54,55)$ and have established that RANKL system components are all present in the rheumatoid synovium. Thus, it appears that local alterations rather than systemic changes of RANKL:OPG ratios are the critical determinants of bone destruction. These data confirm the initial findings in rodent 
adjuvant arthritis models and suggest that RANKL is the principal mediator of bone destruction in human arthritis.

\section{Perspectives}

The association between resorption and the elevation of the RANKL/OPG ratio suggests that the recombinant OPG may be beneficial in a number of conditions. A recent study on postmenopausal women confirmed that OPG reduced bone resorption in vivo: a single monthly injection decreased deoxypyridinoline levels by $80 \%$ (56). OPG might help to combat inflammation-induced bone resorption in patients with RA.

In all experimental animal models studied thus far, inhibition of RANKL had no apparent effect on inflammation, but completely prevented bone loss and partially protected cartilage in all arthritis models studied thus far. The connections between cytokine pro- duction by inflammatory cells and subsequent activation of the RANKL/RANK system point to a unifying paradigm for the entire spectrum of skeletal pathology in RA. Thus, whereas inhibition of TNF and IL-1 using soluble receptor antagonists to some extent prevents inflammation and bone loss in patients with arthritis (57-59), inhibition of the downstream RANKL effectors via OPG or other drugs should prevent bone destruction and cartilage damage in patients with RA irrespective of the initial trigger. Whether inhibition of RANKL will also be beneficial for other forms of arthritis, in particular osteoarthritis, remains to be seen. RANKL inhibition appears to be the most rational and advisable strategy to prevent bone destruction in multiple diseases, to possibly eradicate major human diseases such as osteoporosis, periodontal disease and rheumatoid arthritis that affect millions of people (60).

\section{References}

1. Bromley M \& Wooley DE (1984). Chondrocytes and osteoclasts at subchondral sites of erosions in the rheumatoid joint. Arthritis and Rheumatism, 27: 968-975.

2. Gravallese EM, Manning C, Tsay A, Naito A, Pan C, Amento E \& Goldring SR (2000). Synovial tissue in rheumatoid arthritis is a source of osteoclast differentiation factor. Arthritis and Rheumatism, 43: 250-258.

3. Romas E, Bakharevski O, Hards DK, Kartsogiannis V, Quinn JMW, Ryan PF, Martin TJ \& Gillespie MT (2000). Expression of osteoclast differentiation factor at sites of bone erosion in collagen-induced arthritis. Arthritis and Rheumatism, 43: 821-826.

4. Lacey DL, Timms E, Tan HL et al. (1998). Osteoprotegerin ligand is a cytokine that regulates osteoclast differentiation and activation. Cell, 93: 165-176.

5. Anderson DM, Maraskovsky E, Billingsley WL, Dougall WC Tometsko ME, Roux ER, Teepe MC, DuBose RF, Cosman D \& Galibert L (1997). A homologue of the TNF receptor and its ligand enhance T-cell growth and dendritic-cell function. Nature, 390: 175-179.

6. Wong BR, Rho J, Arron J et al. (1997). TRANCE is a novel ligand of the tumor necrosis factor family that activates c-Jun N-terminal kinase in T-cells. Journal of Biological Chemistry, 272: 2519025194.

7. Lum L, Wong BR, Jasien R, Becherer JD, Erdjument-Bromage $H$, Schlondorff J, Tempst P, Choi Y \& Blobel CP (1999). Evidence for a role of a tumor necrosis factor-alpha (TNF-alpha)-converting enzyme-like protease in shedding of TRANCE, a TNF family mem- ber involved in osteoclastogenesis and dendritic cell survival. Journal of Biological Chemistry, 274: 13613-13618.

8. Kong $Y Y$, Yoshida H, Sarosi I et al. (1999). OPGL is a key regulator of osteoclastogenesis, lymphocyte development and lymph-node organogenesis. Nature, 397: 315-323.

9. Li J, Sarosi I \& Yan XO (2000). RANK is the intrinsic hematopoietic cell surface receptor that controls osteoclastogenesis and regulation of bone mass and calcium metabolism. Proceedings of the National Academy of Sciences, USA, 97: 1566-1571.

10. Fata JE, Kong YY, Li J, Sasaki T, Irie-Sasaki J \& Moorehead RA (2000). The osteoclast differentiation factor osteoprotegerin-ligand is essential for mammary gland development. Cell, 103: 41-50.

11. Hsu, H, Lacey DL, Dunstan CR, Solovyev I, Colombero A \& Timms E (1999). Tumor necrosis factor receptor family member RANK mediates osteoclast differentiation and activation induced by osteoprotegerin ligand. Proceedings of the National Academy of Sciences, USA, 96: 3540-3545.

12. Wong BR, Besser D, Kim N, Arron JR, Vologodskaia M, Hanafusa H \& Choi Y (1999). TRANCE, a TNF family member, activates Akt/PKB through a signaling complex involving TRAF6 and c-Src. Molecular Cell, 4: 1041-1049.

13. Simonet WS, Lancey DL \& Dunstan CL (1997). Osteoprotegerin: a novel secreted protein involved in the regulation of bone density. Cell, 89: 309-319.

14. Bucay N, Sarosi I \& Dunstan CR (1998). Osteoprotegerin-deficient mice develop early onset osteoporosis and arterial calcification. Genes and Development, 12: 1260-1268. 
15. Boyle WJ, Simonet WS \& Lacey DL (2003). Osteoclast differentiation and activation. Nature, 423: 337-342.

16. Josien R, Li HL, Ingulli E, Sarma S, Wong BR, Vologodskaia M, Steinman RM \& Choi $Y$ (2000). TRANCE, a tumor necrosis factor family member, enhances the longevity and adjuvant properties of dendritic cells in vivo. Journal of Experimental Medicine, 191: 495502.

17. Roy M, Waldschmidt T, Aruffo A, Ledbetter JA \& Noelle RJ (1993). The regulation of the expression of gp39, the CD40 ligand, on normal and cloned CD4+ T-cells. Journal of Immunology, 151: 2497-2510.

18. Josien R, Wong BR, Li HL, Steinman RM \& Choi Y (1999). TRANCE, a TNF family member, is differentially expressed on T cell subsets and induces cytokine production in dendritic cells. Journal of Immunology, 162: 2562-2568.

19. Jones DH, Kong YY \& Penninger JM (2002). Role of RANKL and RANK in bone loss and arthritis. Annals of the Rheumatic Diseases, 61: ii-32-ii-39

20. Saika M, Inoue D, Kido S \& Matsumoto T (2001). 17-Beta-estradiol stimulates expression of osteoprotegerin by a mouse stromal cell line, ST-2, via estrogen receptor-alpha. Endocrinology, 142: 22052212.

21. Roggia C, Gao Y, Cenci S, Weitzmann MN, Toraldo G, Isaia G \& Pacifici R (2001). Up-regulation of TNF-producing T-cells in the bone marrow: a key mechanism by which estrogen deficiency induces bone loss in vivo. Proceedings of the National Academy of Sciences, USA, 20: 13960-13965.

22. Goldring SR \& Gravallese EM (2000). Pathogenesis of bone erosions in rheumatoid arthritis. Current Opinion in Rheumatology, 12: 195-199.

23. Saidenberg-Kermanac'h N, Cohen-Solal M, Bessis N, De Vernejoul MC \& Boissier MC (2004). Role of osteoprotegerin in rheumatoid inflammation. Joint Bone Spine, 71: 9-13.

24. Suda T, Takahashi N, Udagawa N, Jimi E, Gillepsie MT \& Martin TJ (1999). Modulation of osteoclast differentiation and function by the new member of the tumor necrosis factor receptor and ligand families. Endocrine Reviews, 20: 345-357.

25. Kotake S, Sato K, Kim KJ, Takahashi N, Udagawa N, Nakamura I, Yamaguchi A, Kishimoto T, Suda T \& Kashiwazaki S (1996). Interleukin-6 and soluble interleukin 6 receptors in the synovial fluids from rheumatoid arthritis patient are responsible for osteoclast-like cell formation. Journal of Bone and Mineral Research, 11: 88-95.

26. Weitzmann MN, Cenci S, Rifas L, Brown C \& Pacifici B (2000). Interleukin-7 stimulates osteoclast formation by up-regulating the T-cell production of soluble osteoclastogenic cytokines. Blood, 96: 1873-1878

27. Kotake S, Udagawa N, Takahashi N et al. (1999). IL-17 in synovial fluids from patients with rheumatoid arthritis is a potent stimulator of osteoclastogenesis. Journal of Clinical Investigation, 103: 13451352.

28. Van Bezooijen RL, Van Der Wee-Pals L, Papapoulos SE \& Lowik CW (2002). Interleukin 17 synergises with tumor necrosis factor alpha to induce cartilage destruction in vitro. Annals of the Rheumatic Diseases, 61: 870-876.

29. Chabaud M \& Miossec $P$ (2001). The combination of tumor necrosis factor alpha blockade with interleukin-1 and interleukin-17 blockade is more effective for controlling synovial inflammation and bone resorption in an ex vivo model. Arthritis and Rheumatism, 44: 1293-1303.

30. Ritchlin CT, Haas Smith SA, Li P, Hicks DG \& Schwarz EM (2003). Mechanisms of TNF-alpha- and RANKL-mediated osteoclastogen- esis and bone resorption in psoriatic arthritis. Journal of Clinical Investigation, 111: 821-831.

31. Takayanagi H, Ogasawara K, Hida S et al. (2000). T-cell-mediated regulation of osteoclastogenesis by signaling cross-talk between RANKL and IFN-gamma. Nature, 408: 600-605.

32. Nakashima T, Wada T \& Penninger JM (2003). RANKL and RANK as novel therapeutic targets for arthritis. Current Opinion in Rheumatology, 15: 280-287.

33. Vidal NO, Brandstrom H, Jonsson KB \& Ohlsson C (1998). Osteoprotegerin mRNA is expressed in primary human osteoblastlike cells: down-regulation by glucocorticoids. Journal of Endocrinology, 159: 191-195.

34. Bendele A, McComb J, Gould T, MaAbee T, Semello G, Chlipala E \& Guy M (1999). Animal models of arthritis: relevance to human disease. Toxicologic Pathology, 27: 134-142.

35. Kong YY, Feige U, Sarosi I et al. (1999). Activated T-cells regulate bone loss and joint destruction in adjuvant arthritis through osteoprotegerin ligand. Nature, 402: 304-309.

36. Campagnuolo G, Bolon B \& Feige U (2002). Kinetics of bone protection by recombinant osteoprotegerin therapy in Lewis rats with adjuvant arthritis. Arthritis and Rheumatism, 46: 1926-1936.

37. Bolon B, Campagnuolo G \& Feige U (2002). Duration of bone protection by a single osteoprotegerin injection in rats with adjuvant-induced arthritis. Cellular and Molecular Life Sciences, 59: 1569-1576

38. Komuro H, Olee T, Kuhn K, Quach J, Brinson DC, Shikhman A, Valbracht J, Creighton-Achermann L \& Lotz M (2001). The osteoprotegerin/receptor activator of nuclear factor kappaB/receptor activator of nuclear factor kappaB ligand system in cartilage. Arthritis and Rheumatism, 44: 2768-2776.

39. Benoist C \& Mathis D (2000). A revival of the B cell paradigm for rheumatoid arthritis pathogenesis? Arthritis Research, 2: 90-94.

40. Kouskoff V, Korganow AS, Duchatelle V, Degott C, Benoist C \& Mathis D (1996) Organ-specific disease provoked by systemic autoimmunity. Cell, 87: 811-822.

41. Pettit AR, Ji H, von Stechow D, Muller R, Goldring SR, Choi $Y$, Benoist C \& Gravallese EM (2001). TRANCE/RANKL knockout mice are protected from bone erosion in the K/BxN serum transfer model of arthritis. American Journal of Pathology, 159: 1689-1699.

42. Ji H, Pettit A, Ohmura K, Ortiz-Lopez A, Duchatelle V, Degott C, Gravallese E, Mathis D \& Benoist C (2002). Critical roles for interleukin 1 and tumor necrosis factor alpha in antibody-induced arthritis. Journal of Experimental Medicine, 196: 77-85.

43. Keffer J, Probert L, Cazlaris H, Georgopoulos S, Kaslaris E, Kioussis D \& Koelias G (1991). Transgenic mice expressing human tumor necrosis factor: a predictive genetic model of arthritis. EMBO Journal, 10: 4025-4031.

44. Redlich K, Hayer S, Maier A et al. (2002). Tumor necrosis factor alpha-mediated joint destruction is inhibited by targeting osteoclasts with osteoprotegerin. Arthritis and Rheumatism, 46: 785792.

45. Schett G, Redllich K, Zwerina J et al. (2003). Osteoprotegerin protects against generalized bone loss in tumor necrosis factortransgenic mice. Arthritis and Rheumatism, 48: 2042-2051.

46. Zwerina J, Hayer S, Tohidast-Akrad M et al. (2004). Single and combined inhibition of tumor necrosis factor, interleukin-1, and RANK pathways in tumor necrosis factor-induced arthritis. Effects on synovial inflammation, bone erosion, and cartilage destruction. Arthritis and Rheumatism, 50: 277-290.

47. Lubberts E, Oppers-Walgreen B, Pettit AR, van den Bersselaar L, Joosten LAB, Goldring SR, Gravallese EM \& van den Berg WB 
(2002). Increase in expression of receptor activator of nuclear factor $\kappa \mathrm{B}$ at sites of bone erosion correlates with progression of inflammation in evolving collagen-induced arthritis. Arthritis and Rheumatism, 46: 3055-3064.

48. Romas E, Sims NA, Hards DK, Lindsay M, Quinn JW, Ryan PF Dunstan CR, Martin TJ \& Gillespie MT (2002). Osteoprotegerin reduces osteoclast numbers and prevents bone erosion in collagen-induced arthritis. American Journal of Pathology, 161: 14191427.

49. Mori H, Kitazawa R, Mizuki S, Nose M, Maeda S \& Kitazawa S (2002). RANK ligand, RANK, and OPG expression in type II collageninduced arthritis mouse. Histochemistry and Cell Biology, 117: 283-292.

50. Kotake S, Udagawa N, Hakoda M et al. (2001). Activated human Tcells directly induce osteoclastogenesis from human monocytes: possible role of T-cells in bone destruction in rheumatoid arthritis patients. Arthritis and Rheumatism, 44: 1003-1012.

51. Ziolkowska M, Kurowska A, Radzikowska A et al. (2002). High levels of osteoprotegerin and soluble receptor activator of nuclear factor kappa B ligand in serum of rheumatoid arthritis patients and their normalization after anti-tumor necrosis factor alpha treatment. Arthritis and Rheumatism, 46: 1744-1753.

52. Haynes DR, Crotti TN, Loric M, Bain GI, Atkins GI \& Findlay DM (2001). Osteoprotegerin and receptor activator of nuclear factor $\kappa B$ ligand (RANKL) regulate osteoclast formation by cells in the human rheumatoid arthritic joint. Rheumatology, 40: 623-630.

53. Takayanagi $H$, lizuka $H$, Juji T, Nakagawa T, Yamamoto A, Miyazaki T, Koshihara Y, Oda H, Nakamura K \& Tanaka S (2000). Involvement of receptor activator of nuclear factor $\kappa B$ ligand/osteoclast differentiation factor in osteoclastogenesis from synoviocyte in rheumatoid arthritis. Arthritis and Rheumatism, 43: 259-269.

54. Itonaga I, Fujikawa Y, Sabokbar A, Murray DW \& Athanasou NA (2000). Rheumatoid arthritis synovial macrophage-osteoclast differentiation is osteoprotegerin ligand-dependent. Journal of $\mathrm{Pa}$ thology, 192: 97-104.

55. Takayanagi H, Oda H, Yamamoto S, Kawaguchi H, Tanaka S, Nishikawa T \& Koshihara $Y$ (1997). A new mechanism of bone destruction in rheumatoid arthritis; synovial fibroblasts induce osteoclastogenesis. Biochemical and Biophysical Research Communications, 240: 279-286.

56. Bekker PJ, Holloway D, Nakanishi A, Arrigh HM, Leese PT \& Dunstan CR (2001). Osteoprotegerin (OPG) has potent and substantial anti-resorptive activity in post menopausal women. Journal of Bone and Mineral Research, 16: 348-360.

57. Fye KH (1999). New treatments for rheumatoid arthritis. Available and upcoming slow-acting antirheumatic drugs. Postgraduate Medicine, 106: 82-85.

58. Graninger WB \& Smolen JS (2001). One-year inhibition of tumor necrosis factor-alpha: a major success or a larger puzzle? Current Opinion in Rheumatology, 13: 9-13.

59. Oliveri MB, Mautalen CA, Rodriguez Fuchs CA \& Romanelli MC (1991). Vertebral compression fractures at the onset of acute lymphoblastic leukemia in a child. Henry Ford Hospital Medical Journal, 39: 45-48.

60. Lerner UH (2004). New molecules in the tumor necrosis factor ligand and receptor superfamilies with importance for physiological and pathological bone resorption. CRC Critical Reviews in Oral Biology and Medicine, 15: 64-81. 